\title{
Antimicrobial and fluoride release capacity of orthodontic bonding materials
}

\author{
Érika Machado CALDEIRA¹, Amanda OSÓRIO', Edna Lúcia Couto OBEROSLER², Delmo Santiago VAITSMAN³ \\ Daniela Sales ALVIANO ${ }^{4}$, Matilde da Cunha Gonçalves NOJIMA ${ }^{5}$
}

\author{
1- DDS, MSD, School of Dentistry, Department of Orthodontics, Federal University of Rio de Janeiro, Rio de Janeiro, RJ, Brazil. \\ 2- Biologist and Technical Chemistry, Institute of Chemistry, Department of Analytical Chemistry, Federal University of Rio de Janeiro, Rio de Janeiro, RJ, Brazil. \\ 3- MSD, PhD, Professor, Institute of Chemistry, Department of Analytical Chemistry, Federal University of Rio de Janeiro, Rio de Janeiro, RJ, Brazil. \\ 4- PhD, Professor, Institute of Microbiology Prof. Paulo de Góes, Federal University of Rio de Janeiro, Rio de Janeiro, RJ, Brazil. \\ 5- MSD, PhD, Associate Professor, School of Dentistry, Department of Orthodontics, Federal University of Rio de Janeiro, Rio de Janeiro, RJ, Brazil.
}

Corresponding address: Matilde da Cunha Gonçalves Nojima - Programa de Pós-Graduação em Odontologia (Ortodontia) - Faculdade de Odontologia Universidade Federal do Rio de Janeiro - Rua Professor Rodolpho Paulo Rocco, nº 325 - Ilha do Fundão - Rio de Janeiro - RJ - Brasil - 21941-617 - Phone: +55 21 2590-2727 - Fax: +55 2125909771 - e-mail: matildenojima@uol.com.br

Submitted: January 17, 2013 - Modification: April 9, 2013 - Accepted: May 17, 2013

\section{ABSTRACT}

\begin{abstract}
bjective: The aim of this study was to evaluate the antimicrobial and fluoride releasing capacity of 3 bonding materials. Material and Methods: Thirty nine specimens with standardized surface smoothness and dimensions were prepared. The antimicrobial capacity of the materials against S. mutans, L. casei and C. albicans was evaluated by determining the percentage of growth inhibition of these microorganisms in an inoculated medium, obtained by optical density readouts on a spectrophotometer. The potential to interfere in microbial growth on the surface of the studied materials was observed by means of scanning electron microscopy (SEM). The fluoride release capacity in ultrapure water for 14 days was analyzed by means of ion chromatography. Results: The PLUS group presented the highest percentage of microbial inhibition and the most contamination-free surface. The FUJI group presented the best fluoride release capacity. Conclusions: The Transbond ${ }^{\mathrm{TM}}$ Plus Color Change was the one that presented the best general behavior considering the evaluated aspects.
\end{abstract}

Key words: Orthodontics. Microbiology. Fluorides. Dental bonding.

\section{INTRODUCTION}

In spite of the various benefits provided by orthodontic treatment, the retentive surfaces of the appliance make them difficult to clean and are directly responsible for food and biofilm accumulation. Imbalances such as gingivitis, gingival hyperplasia and caries may occur during orthodontic treatment ${ }^{6,17,28}$, especially when patients do not cooperate with regard to performing the recommended cleaning protocols. In this context, there is an increase in the acidogenic bacterial populations, such as Streptococcus mutans and Lactobacillus sp in the plaque and saliva 25,28 . The set formed by retentive niches, added to the deficient oral hygiene and a diet rich in fermentable carbohydrates also favors the increase in the Candida albicans population in the oral cavity, particularly if there is caries activity ${ }^{19}$.
There are reports in the literature that microorganisms of the oral microbiome adhere firmly to resin materials. It is also known that components present in the matrix of adhesive materials may accelerate bacterial growth, increasing the levels of pathogens extremely harmful to tissues ${ }^{3}$. Therefore, the ideal bonding material must be capable of withstanding salivary biochemistry, constant changes in $\mathrm{pH}$, different temperatures, and especially the resident oral microbiota. Thus, the importance must be pointed out of maintaining essential properties, such as: hardness, roughness and fluoride release capacity, since these are intimately related to the retention of microorganisms and the maintenance of oral health. In view of the biofilm-composite interaction, which is capable of negatively altering the surfaces of materials for orthodontic bonding, these must have adequate resistance and antimicrobial 
activity $^{3}$.

Fluoridated bonding materials have been inserted outstandingly in Orthodontics because of their fluoride release capacity, irrespective of the patients' cooperation. Nevertheless, it is important to point out that these materials are recommended as supplementary to and not as substitutes for conventional prophylactic alternatives ${ }^{6}$, since their anticariogenic capacity is still being widely discussed ${ }^{2,6,23}$. In view of the context presented, the aim of this study was to evaluate the microbial inhibition capacity of three orthodontic bonding materials, and their fluoride release in vitro. Thus, the hypothesis considered in the present study was that bonding materials play a biological influence to orthodontic treatment outcomes. The capacity of the material to release fluoride and prevent microbial contamination can be noted in the prevention and maintenance of the integrity of the oral tissues.

\section{MATERIAL AND METHODS}

\section{Preparation of specimens}

To conduct the study, three materials extensively applied in bonding orthodontic accessories were selected: Transbond ${ }^{\mathrm{TM}}$ XT (XT Group), a traditional light cure adhesive (3M Unitek, Monrovia, CA, USA), Transbond ${ }^{\mathrm{TM}}$ Plus Color Change (PLUS Group), a moisture tolerant light cure adhesive (3M Unitek, Monrovia, CA, USA) and Fuji Ortho ${ }^{\mathrm{TM}}$ LC (FUJI Group), a resin reinforced glass ionomer cement (GC America Corporation, Tokyo, Japan) (Figure 1). For fabricating the specimens with standardized dimensions, a prefabricated teflon matrix with perforations measuring $5 \mathrm{~mm}$ in diameter and 2 $\mathrm{mm}$ in thickness was used. The materials were manipulated in accordance with the manufacturers' instructions and then inserted into the matrix in a single increment, applying the pressure required for producing specimens with smooth surfaces (Figure 2). Light polymerization was performed for 40 seconds on each surface, using a halogen light with $550 \mathrm{~mW} / \mathrm{cm}^{2}$ (Foto Optilight LD Max, Gnatus - 50/60 Hz, Ribeirão Preto, SP, Brazil), with the active tip of the equipment touching directly on the glass slides ${ }^{26}$.

\section{Microbiologic test}

Microbiologic analyses were performed in two stages. Firstly, the antimicrobial activity of the materials was evaluated by means of spectrophotometry (quantitative aspect). For this, 18 specimens were prepared, divided in duplicate

\begin{tabular}{|c|c|c|c|c|}
\hline Materials & Ingredients & & Manufacturers & Batch \\
\hline $\begin{array}{c}\text { Transbond }{ }^{T M} X T \\
\text { Light Cure Adhesive } \\
\text { (Group XT) }\end{array}$ & $\begin{array}{l}\text { - } \quad \text { Silane treated quartz - 70-80\% } \\
\text { - } \text { Bisphenol A diglycidyl ether dimethacrylate } \\
\text { (BisGMA) - 10-20\% } \\
\text { - } \quad \text { Bisphenol A bis(2-hydroxyethyl ether) } \\
\text { dimethacrylate - } 5-10 \% \\
\text { - } \quad \text { Silane treated silica - }<2 \% \\
\text { - } \quad \text { Diphenyliodonium hexafluorophosphate }-<0.2 \%\end{array}$ & & $\begin{array}{c}\text { 3M Unitek } \\
\text { (Monrovia, USA) }\end{array}$ & N159231 \\
\hline $\begin{array}{c}\text { Transbond }^{\mathrm{TM}} \\
\text { PLUS Color } \\
\text { Change Adhesive } \\
\text { (Group PLUS) }\end{array}$ & 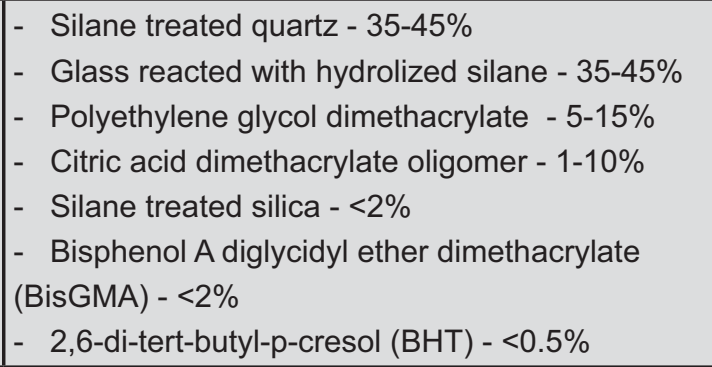 & & $\begin{array}{c}\text { 3M Unitek } \\
\text { (Monrovia, USA) }\end{array}$ & N172805 \\
\hline $\begin{array}{c}\text { Fuji Ortho'TM LC } \\
\text { (Group FUJI) }\end{array}$ & \begin{tabular}{l}
\multicolumn{1}{c}{$\begin{array}{l}\text { Liquid } \\
\text { component }\end{array}$} \\
- $\quad$ Polyacrylic acid - $20-22 \%$ \\
-2 -Hydroxyethyl methacrylate (HEMA) - 35-40\% \\
$-2,2,4$, Trimethyl hexamethylene dicarbonate - \\
$5-7 \%$ \\
$-\quad$ Triethylene glycol dimethacrylate (TEGDMA) - \\
$4-6 \%$ \\
$-\quad$ Proprietary Ingredient - 5-15\%
\end{tabular} & $\begin{array}{c}\text { Powder } \\
\text { component } \\
\text { - Alumino- } \\
\text { silicate glass } \\
-100 \%\end{array}$ & $\begin{array}{l}\text { GC América } \\
\text { Corporation } \\
\text { (Tokyo, Japan) }\end{array}$ & 902121 \\
\hline
\end{tabular}

Figure 1- Groups of materials used in the study and their respective chemical compositions, in accordance with the manufacturers' specifications 


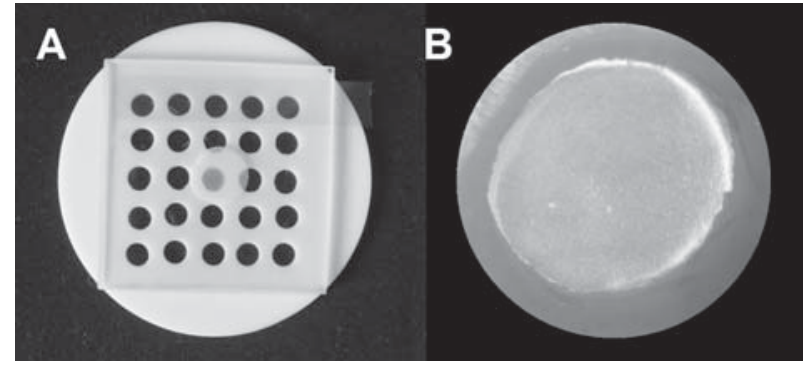

Figure 2- Sequence of specimen preparation: (A) Devices used for fabrication (Teflon matrix, polyester strip and glass slide); (B) Specimen surfaces with smoothness and mirror brightness obtained immediately after removal from the matrix

for each tested microorganism.

With regard to the qualitative aspect of analyses, after fabricating one control specimen for each material (baseline), the initial surface (contamination-free) record was made by scanning electron microscopy (SEM). After evaluation on the spectrophotometer, one specimen per microorganism was randomly selected from each group, to be submitted to a SEM analysis to verify microbial growth on its surfaces after contamination, and therefore, the inherent antimicrobial potential of each material. For each specimen evaluated by the SEM, various images were obtained in order to contemplate a large scanning area.

\section{Quantitative analysis of antimicrobial activity}

The following strains were used in the experiment: S. mutans (ATCC 25175), L. casei (ATCC 4646) and C. albicans (ATCC 10231), kept in BHI broth (Brain Heart Infusion, Himedia, São Paulo, SP, Brazil). Initially, the BHI broth contaminated with the microorganisms of choice (positive control) was placed into 24 well plates, interspersed with wells containing sterile medium (negative control) and inoculated medium in conjunction with the specimens that would have their antimicrobial capacity tested. The quantity of cells in the experiment was standardized in order to attain the desired concentration of $5 \times 10^{3}$ cells per well $(500 \mu \mathrm{l})$. The plates were incubated in an oven at $37^{\circ} \mathrm{C}$, and the plate with $S$. mutans, was placed in an anaerobic jar. After $24 \mathrm{~h}$, the optical density (OD) readout of each well was taken, starting by calibrating the spectrophotometer to $550 \mathrm{~nm}$ (Beckman Coulter DU 530 Spectrophotometer, Fullerton, CA, USA) by taking the sterile medium readout. The inoculated medium readout consisted of the positive control of growth for each microorganism ${ }^{1,13}$.

To obtain the percentage of microbial growth inhibition of the different groups studied, the following equation was used, with: $(M)$ mean of optical densities of each material tested; (C) positive control of growth: \% growth inhibition $=100-(\mathrm{M} / \mathrm{C}) \times 100$.

Qualitative analysis of antimicrobial activity

In addition to quantifying the antimicrobial activity by spectrophotometry, the specimens were analyzed by SEM, in order to visualize the microbial proliferation on the surface of each studied material. To obtain the initial record of the material surfaces (baseline), one specimen from each group was prepared on aluminum stubs, and gold sputtered for examination by scanning electron microscopy (JEOL-JSM; 5800LV, Tokyo, Japan). A voltage of $30 \mathrm{kV}$ and low vacuum mode (45 Pa) was used. Immediately after the quantitative analysis of the antimicrobial activity, one specimen per microorganism from each group was randomly selected and fixed stored in $2.5 \%$ glutaraldehyde and $0.1 \mathrm{M}$ cacodylate buffer. After $2 \mathrm{~h}$, the specimens were removed, washed with PBS (phosphate buffered saline) solution and dried. Next, they were gold sputtered to obtain the final records under conditions similar to the initial ones. Various records of each specimen were obtained with the goal of extensive scanning of the surface. The images were obtained at 5000x magnification.

\section{Fluoride release analysis}

For the fluoride release analysis, 6 specimens were prepared for each group (XT, PLUS, FUJI), totaling 18 specimens. After each specimen was removed from the teflon matrix, its base and lateral surfaces were sealed with sticky wax, and it was adequately identified, thus only the surface area of $19.62 \mathrm{~mm}^{2}$ remained exposed. For each specimen, 15 tubes of the Eppendorf ${ }^{\circledR}$ type, previously washed for ionic decontamination, were filled with $1.5 \mathrm{~mL}$ of ultrapure water. The Eppendorf ${ }^{\circledR}$ tubes corresponded to the 15 predetermined time intervals of fluoride release, with variation from 1 hour to the $14^{\text {th }}$ day, identified as follows: $1 \mathrm{~h}, 1 \mathrm{~d}$, $2 \mathrm{~d}, 3 \mathrm{~d}, 4 \mathrm{~d}, 5 \mathrm{~d}, 6 \mathrm{~d}, 7 \mathrm{~d}, 8 \mathrm{~d}, 9 \mathrm{~d}, 10 \mathrm{~d}, 11 \mathrm{~d}, 12$ $\mathrm{d}, 13 \mathrm{~d}$ and $14 \mathrm{~d}$. During this cycle, the tubes were kept in a microbiologic oven at $37^{\circ} \mathrm{C}$, separately in receptacles which were named XT, PLUS and FUJI.

The daily time when the specimens were moved into their new tubes was standardized. The specimens were manipulated with tweezers and they had been delicately dried with absorbent paper before they were reinserted into the sequence of the cycle. As the changes occurred, the solutions in which the specimens had been immersed were sent for laboratory analysis. It should be pointed out that the ultrapure water was the renewed vehicle, since the same specimen was kept from the beginning to the end of the experiment. The purpose of this 
was to identify the daily fluoride release capacity, by exhaustion of this same material throughout the cycle. During the experiment, no ionic supplement whatsoever was inserted. The ultrapure water was renewed in an attempt to eliminate any possible saturation of the medium.

This study generated a total of 270 accumulated fluoride release readouts, performed by ion Chromatography (DX 80 Ion Analyzer, Dionex, Sunnyvale, CA, USA), in order to perform the separation and determination of fluoride content in each solution. Before the readouts, the ion Chromatograph was calibrated with 5 specific patterns, these being: 1-ultrapure water; 2- pattern 1 ( $\mathrm{F}^{-}$concentration $\left.=0.02 \mathrm{mg} / \mathrm{L}\right)$; 3pattern $2\left(\mathrm{~F}^{-}\right.$concentration $\left.=0.2 \mathrm{mg} / \mathrm{L}\right) ; 4$ - pattern $3\left(\mathrm{~F}^{-}\right.$concentration $\left.=1.0 \mathrm{mg} / \mathrm{L}\right) ; 5$ - pattern 4 $\left(\mathrm{F}^{-}\right.$concentration $\left.=2.0 \mathrm{mg} / \mathrm{L}\right)$. The data obtained were transferred and processed by an integrator, automatic module Dionex 4400 that promotes complete automation of the system. The time that elapsed for specific determination of the desired ion was approximately 12 minutes for each $1.5 \mathrm{~mL}$ sample of ultrapure water.

\section{Statistical analysis}

The percentage of growth inhibition data for each specimen of the XT, PLUS and FUJI groups obtained in the microbiologic analysis, as well as the fluoride release tests, were submitted to the analysis of variance ANOVA and Tukey Test in the SPSS 17.0 software (Statistical Package for Social Sciences, SPSS Inc., Chicago, IL, USA). The difference between the means was considered significant when values of $p<0.05$ were obtained.

The results obtained for the qualitative analysis of antimicrobial capacity were descriptively recorded

\section{RESULTS}

\section{Quantitative analysis of antimicrobial} activity

Table 1 shows the results obtained in the microbiologic test, specifying the activity of the materials in relation to the microorganisms analyzed, with respect to the microbial growth and inhibition.

To evaluate the microbiologic action of materials against $S$. mutans and $L$. casei, it was shown that only the PLUS presented a significant reduction in growth $(p<0.05)$. With regards to action against C. albicans, the materials showed significant differences among them $(p<0.05)$, with emphasis on the PLUS, since it was the material that presented significant inhibition potential against the fungus $(p<0.001)$.

\section{Qualitative analysis of antimicrobial activity}

The SEM photomicrographs shown in Figure $3(1 \mathrm{~A}, 2 \mathrm{~A}, 3 \mathrm{~A})$ reveal the images of the negative control specimen surfaces from the $X T$, PLUS and FUJI groups, respectively. Images $1 \mathrm{~B}$ and 3B denote $S$. mutans growth on XT and FUJI specimens, in this order. Visually, the greatest microbial growth occurred in the FUJI group (3B), whereas the PLUS group was found to have a contamination-free surface (2B), which also occurred in image $2 C$, in which no $L$. casei was observed on the surface of the specimen from the PLUS group. Images $1 \mathrm{C}$ (XT) and $3 \mathrm{C}$ (FUJI) also point out microbial growth, which was more expressive in the FUJI group. Lastly, it is possible to visualize great $C$. albicans proliferation on the

Table 1- Results of microbiologic analysis of specimens, specifying: Mean optical densities (OD) of positive controls for each group of microorganisms (OD control), values obtained in duplicate for each material tested (OD1, OD2), mean and standard deviation (SD), and percentage of microbial growth inhibition (\%)

\begin{tabular}{ccccccc}
\hline S. mutans & OD control & OD1 & OD2 & Mean & SD & Inhibition \\
XT & & 0.377 & 0.448 & 0.412 & 0.050 & $28.70 \%^{\mathrm{B}}$ \\
PLUS & 0.578 & 0.012 & 0.005 & 0.008 & 0.004 & $98.60 \%^{\mathrm{A}}$ \\
FUJI & & 0.456 & 0.518 & 0.487 & 0.043 & $15.80 \%^{\mathrm{B}}$ \\
\hline L. casei & OD control & OD1 & OD2 & Mean & SD & Inhibition \\
XT & & 0.443 & 0.248 & 0.345 & 0.137 & $45.10 \%^{\mathrm{B}}$ \\
PLUS & 0.628 & 0.000 & 0.000 & 0.000 & 0.000 & $100 \%^{\mathrm{A}}$ \\
FUJI & & 0.488 & 0.501 & 0.494 & 0.009 & $21.30 \%^{\mathrm{B}}$ \\
\hline C. albicans & OD control & OD1 & OD2 & Mean & SD & Inhibition \\
XT & & 0.473 & 0.483 & 0.478 & 0.007 & $15.70 \%^{\mathrm{B}}$ \\
PLUS & 0.567 & 0.000 & 0.004 & 0.002 & 0.002 & $99.70 \%^{\mathrm{A}}$ \\
FUJI & & 0.522 & 0.549 & 0.535 & 0.019 & $5.60 \%^{\mathrm{C}}$ \\
\hline
\end{tabular}

Different letters express statistically significant values $(p<0.05)$, being $A>B>C$ 


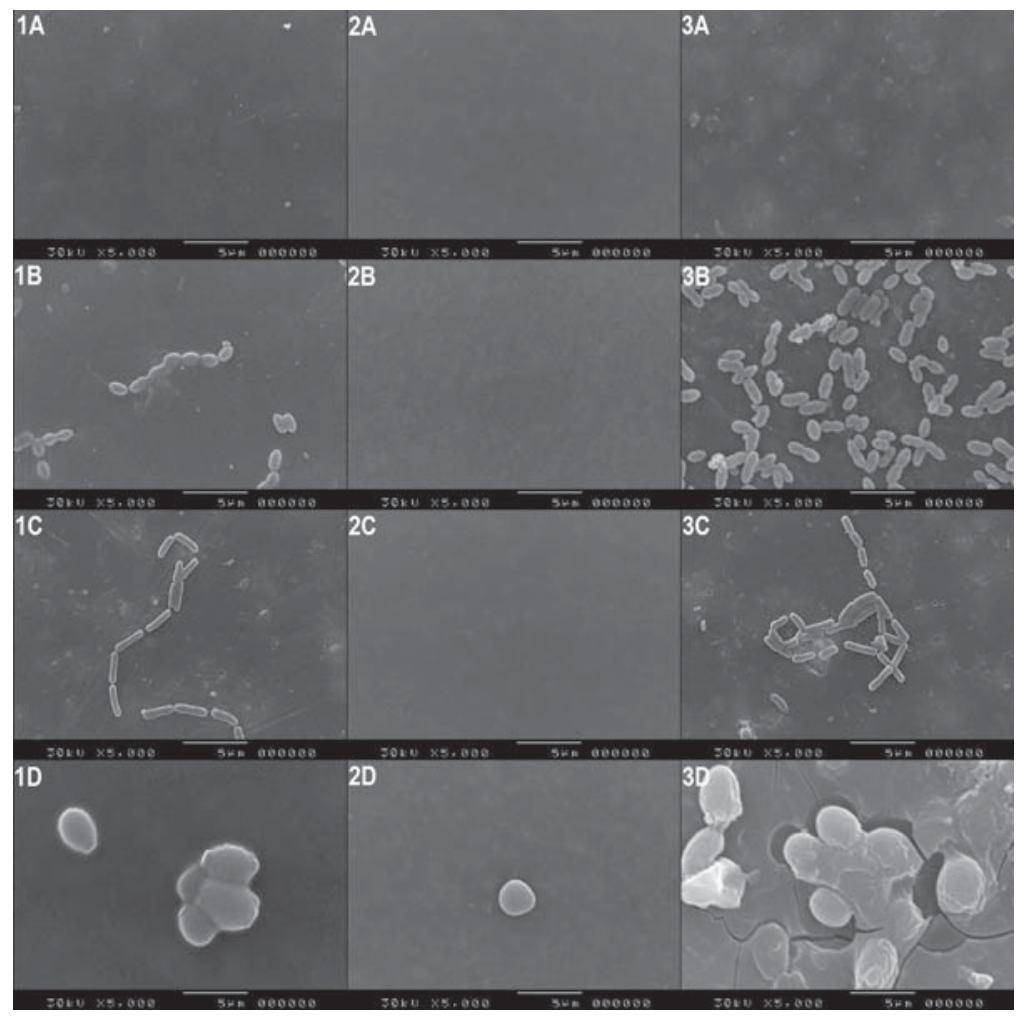

Figure 3- Scanning electron microscopy photomicrographs showing the surfaces of tested materials: (1) XT Group, (2) PLUS Group and (3) FUJI Group (columns); (A) Baseline, negative control, (B) S.mutans, (C) L.casei and (D) C.albicans (lines)

Table 2- Mean* (standard deviation) of fluoride release in ppm of the bonding materials $(n=6)$ during the 15 time intervals

\begin{tabular}{|c|c|c|c|c|c|c|c|c|c|c|c|c|c|c|c|}
\hline F- & $1 \mathrm{~h}$ & $1 \mathrm{~d}$ & $2 d$ & $3 \mathrm{~d}$ & $4 \mathrm{~d}$ & $5 \mathrm{~d}$ & $6 \mathrm{~d}$ & $7 d$ & $8 d$ & $9 \mathrm{~d}$ & $10 \mathrm{~d}$ & $11 \mathrm{~d}$ & $12 d$ & $13 \mathrm{~d}$ & $14 \mathrm{~d}$ \\
\hline \multirow[t]{2}{*}{$\overline{X T}$} & $0.007^{c}$ & $0.004^{c}$ & $0.001^{c}$ & $0.000^{c}$ & $0.002^{c}$ & $0.001^{\mathrm{c}}$ & $0.001^{c}$ & $0.001^{c}$ & $0.002^{c}$ & $0.002^{c}$ & $0.002^{B}$ & $0.002^{c}$ & $0.001^{\mathrm{c}}$ & $0.001^{c}$ & $0.002^{\mathrm{C}}$ \\
\hline & $(0.005)$ & $(0.002)$ & $(0.001)$ & $(0.000)$ & $(0.001)$ & $(0.001)$ & $(0.000)$ & $(0.002)$ & (0.001) & $(0.002)$ & $(0.002)$ & $(0.001)$ & (0.001) & (0.001) & $(0.001)$ \\
\hline \multirow[t]{2}{*}{ PLUS } & $0.087^{\mathrm{B}}$ & $1.007^{\mathrm{B}}$ & $0.628^{\mathrm{B}}$ & $0.465^{\mathrm{B}}$ & $0.440^{B}$ & $0.428^{\mathrm{B}}$ & $0.424^{\mathrm{B}}$ & $0.446^{\mathrm{B}}$ & $0.448^{\mathrm{B}}$ & $0.618^{\mathrm{B}}$ & $0.609^{A}$ & $0.583^{B}$ & $0.502^{\mathrm{B}}$ & $0.479^{\mathrm{B}}$ & $0.454^{\mathrm{B}}$ \\
\hline & $(0.029)$ & $(0.420)$ & $(0.750)$ & $(0.876)$ & $(0.032)$ & $(0.024)$ & $(0.058)$ & $(0.187)$ & $(0.163)$ & $(0.105)$ & $(0.106)$ & $(0.097)$ & $(0.070)$ & (0.089) & (0.077) \\
\hline \multirow[t]{2}{*}{ FUJI } & $0.267^{A}$ & $2.564^{\mathrm{A}}$ & $1.276^{\mathrm{A}}$ & $1.129^{A}$ & $1.031^{\mathrm{A}}$ & $1.106^{A}$ & $0.839^{A}$ & $0.829^{A}$ & $0.938^{A}$ & $0.908^{A}$ & $0.691^{\mathrm{A}}$ & $0.780^{A}$ & $0.632^{A}$ & $0.634^{\mathrm{A}}$ & $0.845^{\mathrm{A}}$ \\
\hline & $(0.087)$ & $(0.821)$ & $(0.293)$ & $(0.309)$ & $(0.193)$ & $(0.260)$ & $(0.152)$ & $(0.221)$ & $(0.190)$ & $(0.125)$ & $(0.148)$ & $(0.094)$ & $(0.128)$ & $(0.090)$ & $(0.121)$ \\
\hline
\end{tabular}

* Means followed by different letters differ statistically at the level of significance of 0.05 (comparison in column for the same time analyzed, being $A>B>C$ ).

FUJI surface (3D), followed by XT (1D) and PLUS (2D), although insignificantly on the latter.

\section{Fluoride release analysis}

The fluoride release data during the 14 days of the experiment are shown in Table 2, and are expressed comparatively in Figure 4. One observes that the FUJI was the most outstanding material for fluoride release, especially in the first 24 hours, followed by the PLUS and XT, respectively. Although the latter characteristically does not release fluoride, the rates found were very low and insignificant, being directly related to the accuracy of the technique. On the tenth day of the cycle, the values obtained for the FUJI and PLUS were similar $(p>0.05)$.

\section{DISCUSSION}

There has been much discussion about the maintenance of the oral health in patients submitted to orthodontic treatment, such as the formation of initial caries lesions, which even today, is a potential risk. In a study recently conducted with 230 individuals, $71.7 \%$ developed from 1 to 12 white spot lesions at the end of treatment, and $69.8 \%$ presented adequate oral hygiene conditions at the beginning of orthodontic therapy ${ }^{17}$. These data are indicative of the importance of instituting a good preventive program in these patients. Should there be clinical signs of disease progression, efforts must be redoubled in an attempt to 


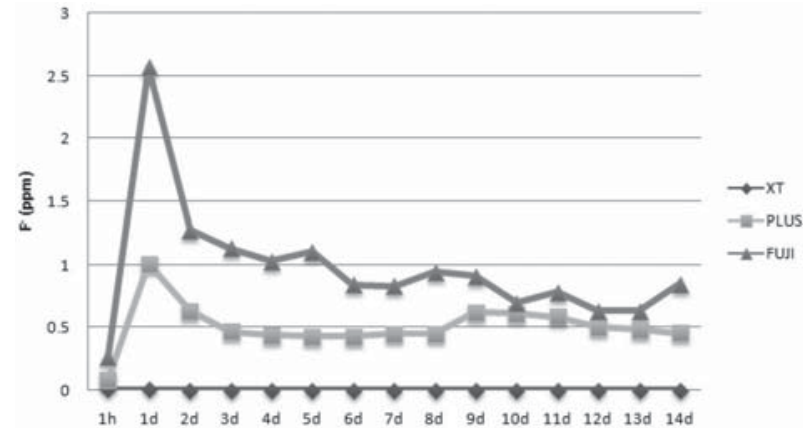

Figure 4- Quantity of fluoride in ppm released by each material during the time intervals evaluated throughout the study

revert the situation of demineralization. Thus, it is possible to use materials that have fluoride in their composition, with the purpose of helping to prevent caries development. Nevertheless, the action of fluoride as an antibacterial agent is still widely discussed, in spite of it clearly having remineralization potential ${ }^{24}$.

The results obtained demonstrated the greater efficacy of the Transbond ${ }^{\mathrm{TM}}$ Plus Color Change as an antimicrobial material, since it showed the best percentages of growth inhibition for all the species evaluated. The Fuji Ortho ${ }^{\mathrm{TM}}$ LC presented the most unfavorable result with regards to antimicrobial capacity, however, it showed the greatest fluoride release, which raises questions about the direct relationship of fluoride with microbial growth inhibition. If only fluoride release was responsible for the antimicrobial potential of the material, the FUJI, which released the most fluoride in comparison with the other groups with regards to antimicrobial activity, which was not demonstrated in the findings of this research.

Considering physicochemical characteristics ${ }^{10}$, Lee, et al. ${ }^{16}$ (2009) in their study, concluded that there is greater microbial adherence to the surfaces of the bonding materials than to the orthodontic appliance accessories, and associated this fact with the free surface energy of these materials. Greater adhesion of S. mutans to the Fuji Ortho ${ }^{\mathrm{TM}}$ LC was also found than on the composites, corroborating the quantitative and qualitative findings of the study. It is also known that the surface roughness of materials is directly proportional to the increase in microbial adhesion ${ }^{5,20}$. Therefore, the fact that glass ionomer cement is a material with low resistance and greater roughness, when compared with composites, also justifies the behavior of the FUJI in the present study. With regard to chemical composition, the Fuji Ortho ${ }^{\mathrm{TM}}$ LC contains the co-monomer TEGDMA, described in the literature as being capable of stimulating microbial proliferation $^{13,15,18}$.

Authors have described the main characteristics of ionomer materials as being anticariogenic and having antimicrobial properties. The former, is directly related to the preventive and remineralizing potential of fluoride, whereas the latter is associated with the presence of fluoride and aluminum ${ }^{14}$. However, in this study, neither the fluoride nor aluminum of the Fuji Ortho ${ }^{\mathrm{TM}} \mathrm{LC}$ was shown to be efficient in contributing to microbial reduction and adhesion on its surface. This denotes the prevalence of the other previously described aspects of these possible benefits.

Factors such as the initial $\mathrm{pH}$ of the material, hydrophobicity and chemical substances released are also related to antimicrobial activity ${ }^{10,14}$. Chin, et al. ${ }^{10}$ (2006) studied the hydrophobicity of some orthodontic bonding materials, among them the Transbond ${ }^{\mathrm{TM}} \mathrm{XT}$ and Fuji Ortho ${ }^{\mathrm{TM}} \mathrm{LC}$, both demonstrating similar hydrophobicity values, and representing the most expressive values in the study. Consequently, the greatest biofilm formation was found in these materials. Similar results were found by Ahn, et al. ${ }^{1}$ (2009), who observed that both the Transbond ${ }^{\mathrm{TM}} \mathrm{XT}$ and Fuji Ortho ${ }^{\mathrm{TM}}$ LC exercised no antimicrobial activity, with the FUJI having shown the greatest microbial proliferation, corroborating the findings of the present study.

Although the cytotoxicity of the material may be related to microbial growth inhibition, it cannot be taken as the only justification for the results found in this research, because all the materials tested released substances such as bisphenol $A$, Bis-EMA, EGDMA, TEGDMA, HEMA, among others, capable of promoting adverse biologic reactions ${ }^{15,18}$. Therefore, it would not be conclusive to attribute the bests results found for the PLUS exclusively to cytotoxicity.

With respect to fluoride release, the majority of studies with the goal of quantifying it use the ionselective electrode in their methodology ${ }^{2,7}$, since there is a scarcity of the use of ion chromatography in the literature to proceed with such an analysis. This technique is frequently used when one desires to obtain the other components in the sample, because of its efficient ion detector system. In the present study, the chosen methodology for measuring fluoride ions was shown to be adequate and to have excellent precision because it determined anions and cations at trace levels ${ }^{4}$.

During the 15 time intervals of analysis for fluoride quantification, in addition to the classic increase in release in the $24 \mathrm{~h}^{7}$, there were some moments of slight increase in fluoride release, but no continually decreasing release was verified with the passage of the time considered (Figure 4). Reduction in the quantity of fluoride is explained by the fact that the components of the external layer of the material became exhausted, and were dissolved in the water. With regard to the PLUS 
group, the release of fluoride contained within the matrix is a little limited by the resin components that make it difficult to displace. However, since the matrix of the FUJI is more sensitive to hydration, there is less limitation of ion displacement ${ }^{8}$. This justifies the higher values in this group, as well as the more recurrent peaks, in spite of it having been equivalent to the PLUS on the $10^{\text {th }}$ day of the cycle.

It is possible to associate the initial fluoride release from the surface and the later release from the subsurface of the material matrix. The initial analog being a "superficial washing", whereas the release afterwards, said to be late, is allowed through the micropores and from the matrix of the material itself 7,11 .

Other studies have also verified a small quantity of fluoride detectible in the Transbond ${ }^{\mathrm{TM}} \mathrm{XT}$, and the better performance of the Fuji Ortho ${ }^{\mathrm{TM}} \mathrm{LC}$ when compared with the Transbond ${ }^{\mathrm{TM}}$ Plus Color Change. The greater fluoride release from resinmodified glass ionomer cements (RMGIC) is attributed to the acid-base reaction between the aluminosilicate glass powder containing fluoride and polyacid liquid, which results in the release of fluoride ions ${ }^{2,7}$. The porosity of the material also has a strong relationship with the quantity of fluoride released, and the greater fluoride release by the FUJI in comparison with the composites is therefore justifiable ${ }^{29}$. Ahn, et al. $^{2}$ (2011) observed that RMGICs tend to have greater fluoride ion release and recharge capacity, and these ions penetrate precisely into the spaces previously occupied by the fluoride that was released. It is important to emphasize that the process of matrix erosion is directly related to the diffusion of ions and fluoride release ${ }^{27}$.

In spite of the more expressive fluoride release occurring in the first few hours, one could attribute the rapid formation of calcium fluoride on the enamel surface to this factor, which is of clinical importance in protecting the tooth against demineralization ${ }^{2,7,21}$. In a previous study by Gorton and Featherstone ${ }^{12}$ (2003), it was proved that irrespective of the antimicrobial potential, the fluoride contained in the Fuji Ortho ${ }^{\mathrm{TM}}$ LC, used for the bracket bonding, was capable of promoting a cariostatic effect in vivo, when compared with the Transbond ${ }^{\mathrm{TM}} \mathrm{XT}$.

In view of the above discussion, the presence of fluoride available to patients submitted to orthodontic treatment is of extreme importance in preventing the appearance of caries lesions ${ }^{9}$, highlighting the bonding material capable of exercising this function ${ }^{22}$. In a similar manner, the interesting factor with regards to the antimicrobial activity of the material also refers to the protection against its surface degradation. The material capable of reducing microbial growth, and particularly microorganism adhesion on its surface, is able to prevent a cycle of deterioration, since bacterial growth on the material is capable of increasing its roughness and accelerating biofilm accumulation ${ }^{3}$.

\section{CONCLUSION}

This study supports the hypothesis that bonding materials can contribute positively to orthodontic treatment outcomes, with an essential role in the maintenance of health and balance in the oral cavity. The importance is emphasized of the material being able to withstand microbial attacks in order to maintain its integrity and that of the adjacent tissues. In this respect, the Transbond ${ }^{\mathrm{TM}}$ Plus Color Change was outstanding among the others, and the Fuji Ortho ${ }^{\mathrm{TM}}$ LC presented less satisfactory results. However, with regard to the fluoride release capacity, the FUJI group guaranteed more expressive results, emphasizing its relevant role in anticariogenic activity. Therefore, one must consider that there is no ideal material, and the choice should be based on scientific findings and clinical experience, respecting the individuality of each case.

\section{REFERENCES}

1- Ahn S, Lee S, Kook J, Lim B. Experimental antimicrobial orthodontic adhesives using nanofillers and silver nanoparticles. Dent Mater. 2009;25:206-13.

2- Ahn SJ, Lee SJ, Lee DY, Lim BS. Effects of different fluoride recharging protocols on fluoride ion release from various orthodontic adhesives. J Dent. 2011;39:196-201.

3- Beyth N, Bahir R, Matalon S, Domb AJ, Weiss EI. Streptococcus mutans biofilm changes surface-topography of resin composites. Dent Mater. 2008;24:732-6.

4- Biemer TA, Asral N, Sippy A. Ion chromatographic procedures for analysis of total fluoride content in dentifrices. J Chromatogr A. $1997 ; 771: 355-9$.

5- Bollen CM, Lambrechts P, Quirynen M. Comparison of surface roughness of oral hard materials to the threshold surface roughness for bacterial plaque retention: a review of the literature. Dent Mater. 1997;13:258-69.

6- Büyükyilmaz T, Øgaard B. Caries-preventive effects of fluoridereleasing materials. Adv Dent Res. 1995;9:377-83.

7- Cacciafesta V, Sfondrini MF, Tagliani P, Klersy C. In-vitro fluoride release rates from 9 orthodontic bonding adhesives. Am J Orthod Dentofacial Orthop. 2007;132:656-62.

8- Calabrich CFC, Barbosa MC, Simionato MRL, Ferreira RFA. Evaluation of antimicrobial activity of orthodontic adhesive associated with chlorhexidine-thymol varnish in bracket bonding. Dental Press J Orthod. 2010;15:62-8.

9- Caldeira EM, Fidalgo TKS, Passalini P, Marquezan M, Maia LC, Nojima MCG. Effect of fluoride on tooth erosion around orthodontic brackets. Braz Dent J. 2012;23:581-85.

10- Chin MYH, Sandham A, Pratten J, De Vries J, Van der Mei HC, Busscher $\mathrm{HJ}$. Multivariate analysis of surface physico-chemical properties controlling biofilm formation on orthodontic adhesives prior to and after fluoride and chlorhexidine treatment. J Biomed Mater Res Part B Appl Biomater. 2006;78B:401-8. 
11- Gandolfi MG, Chersoni S, Acquaviva GL, Piana G, Prati C, Mongiorgi R. Fluoride release and absorption at different $\mathrm{pH}$ from glass-ionomer cements. Dent Mater. 2006;22:441-9.

12- Gorton J, Featherstone J. In vivo inhibition of demineralization around orthodontic brackets. Am J Orthod Dentofacial Orthop. 2003; $123: 10-4$

13- Hansel C, Leyhausen G, Mai UE, Geurtsen W. Effects of various resin composite ( $\mathrm{Co}$ )monomers and extracts on two cariesassociated micro-organisms in vitro. J Dent Res. 1998;77:60-7. 14- Hayacibara MF, Rosa OP, Koo H, Torres AS, Costa B, Cury JA. Effects of fluoride and aluminum from ionomeric materials on $S$. mutans biofilm. J Dent Res. 2003;82:267-71.

15- Huang TH, Tsai CY, Chen SL, Kao CT. An evaluation of the cytotoxic effects of orthodontic bonding adhesives upon a primary human oral gingival fibroblast culture and a permanent, human oral cancer-cell line. J Biomed Mater Res Part B Appl Biomater. 2002;63:814-21.

16- Lee SP, Lee SJ, Lim BS, Ahn SJ. Surface characteristics of orthodontic materials and their effects on adhesion of mutans streptococci. Angle Orthod. 2009;79:353-60.

17- Maaitah EFA, Adeyemi AA, Higham SM, Pender N, Harrison JE. Factors affecting demineralization during orthodontic treatment: a post-hoc analysis of RCT recruits. Am J Orthod Dentofacial Orthop. 2011;139:181-91.

18- Malkoc S, Corekci B, Ulker HE, Yalçin M, Sengün A. Cytotoxic effects of orthodontic composites. Angle Orthod. 2010;80:759-64 19- Mardegan RC, Klein MI, Golvea MB, Rodrigues JAO, Gonçalves RB, Höfling JF. Biotyping and genotypic diversity among oral Candida albicans strains from caries-free and caries-active healthy children. Braz J Microbiol. 2006;37:26-32.

20- Mei L, Busscher HJ, van der Mei HC, Ren Y. Influence of surface roughness on streptococcal adhesion forces to composite resins. Dent Mater. 2011;27:770-8.
21- Millett DT, Nunn JH, Welbury RR, Gordon PH. Decalcification in relation to brackets bonded with glass ionomer cement or a resin adhesive. Angle Orthod. 1999;69:65-70.

22- Passalini P, Fidalgo TKS, Caldeira EM, Gleiser R, Nojima MCG, Maia LC. Mechanical properties of one and two-step fluoridated orthodontic resins submitted to different $\mathrm{pH}$ cycling regimes. Braz Oral Res. 2010;24:197-203.

23- Passalini P, Fidalgo TKS, Caldeira EM, Gleiser R, Nojima MCG, Maia LC. Preventive effect of fluoridated orthodontic resins subjected to high cariogenic challenges. Braz Dent J. 2010;21:211-5.

24- Sanders BJ, Gregory RL, Moore K, Avery DR. Antibacterial and physical properties of resin modified glass-ionomers combined with chlorhexidine. J Oral Rehabil. 2002;29:553-8.

25- Sanpei S, Endo T, Shimooka S. Caries risk factors in children under treatment with sectional brackets. Angle Orthod. 2010;80:509-14.

26- Silva KG, Pedrini D, Delbem ACB, Cannon M. Effect of $p H$ variations in a cycling model on the properties of restorative materials. Oper Dent. 2007;32:328-35.

27- Silva KG, Pedrini D, Delbem AC, Cannon M. Microhardness and fluoride release of restorative materials in different storage media. Braz Dent J. 2007;18:309-13.

28- Tufekci E, Dixon JS, Gunsolley JC, Lindauer SJ. Prevalence of white spot lesions during orthodontic treatment with fixed appliances. Angle Orthod. 2011;81:206-10.

29- Xu X, Burgess JO. Compressive strength, fluoride release and recharge of fluoride-releasing materials. Biomaterials. $2003 ; 24: 2451-61$ 